\title{
Initial Abstract
}

for STAIF - 2007

\section{Microwave Extraction of Water from Lunar Regolith Simulant}

\author{
Edwin Ethridge, NASA, Marshall Space Flight Center, EM40 \\ William Kaukler, University of Alabama, Huntsville
}

\begin{abstract}
Nearly a decade ago the DOD Clementine lunar orbital mission obtained data indicating that the permanently shaded regions at the lunar poles may have permanently frozen water in the lunar soil. Currently NASA's Robotic Lunar Exploration Program, RLEP-2, is planned to land at the lunar pole to determine if water is present. The detection and extraction of water from the permanently frozen permafrost is an important goal for NASA. Extraction of water from lunar permafrost has a high priority in the In-Situ Resource Utilization, ISRU, community for human life support and as a fuel. The use of microwave processing would permit the extraction of water without the need to dig, drill, or excavate the lunar surface. Microwave heating of regolith is potentially faster and more efficient than any other heating methods due to the very low thermal conductivity of the lunar regolith. Also, microwaves can penetrate into the soil permitting water removal from deep below the lunar surface. A cryogenic vacuum test facility was developed for evaluating the use of microwave heating and water extraction from a lunar regolith permafrost simulant. Water is obtained in a cryogenic cold trap even with soil conditions below $0^{\circ} \mathrm{C}$. The results of microwave extraction of water experiments will be presented.
\end{abstract}




\title{
Microwave Extraction of Water from Lunar Regolith Simulant
}

\author{
Edwin Ethridge ${ }^{1}$ and William Kaukler ${ }^{2}$
}

${ }^{l} N A S A-M S F C-E M 40$

Huntsville, $A L 35812$

256-544-7767

Ed.Ethridge@nasa.gov

${ }^{2}$ Department of Chemistry

301 Sparkman Ave.

University of Alabama, in Huntsville

Huntsville, AL 35899

William.Kaukler@msfc.nasa.gov

\begin{abstract}
.
Nearly a decade ago the DOD Clementine lunar orbital mission obtained data indicating that the permanently shaded regions at the lunar poles may have permanently frozen water in the lunar soil. Currently a Lunar Lander Exploration Program, is expected to land at the lunar pole to determine if water is present. The detection of water from the permanently frozen permafrost is an important goal for NASA. Extraction of water from lunar permafrost would be a valuable In-Situ Resource for Utilization (ISRU) in human life support and as a fuel. The use of microwave processing could permit the extraction of water without the need to dig, drill, or excavate the lunar surface. Microwave heating of regolith is potentially faster and more efficient than any other heating methods due to the very low thermal conductivity of the lunar regolith. Also, microwaves can penetrate into the soil permitting water removal from deep below the lunar surface. A cryogenic vacuum test facility was developed for evaluating the use of microwave heating and water extraction from a lunar regolith permafrost simulant. Water was collected in a cryogenic cold trap even with soil temperature well below $0^{\circ} \mathrm{C}$. The results of microwave extraction of water experiments will be presented.
\end{abstract}

Keywords: lunar water, lunar regolith simulant, microwave processing, lunar experiments

PACS: $84.40 .-\mathrm{x}, 95.55 . \mathrm{Pe}, 78.70 . \mathrm{Gq}, 77.22 . \mathrm{d}$ 


\section{INTRODUCTION}

The objective of this research is to determine the effectiveness of using microwave energy to extract water from cryogenically cold lunar regolith soil simulant. Water and a number of other useful volatiles possibly exist on the moon and are said to be concentrated in the permanently shaded craters in the Polar regions. Evidence of lunar water ${ }^{1}$ was indicated by remote sensing from the Clementine Lunar orbiter with the Bistatic Radar Experiment. Lunar Prospector, the orbiting Neutron Spectrometer showed as much as $4 \%$ water-ice in the shaded poles to a depth of $1 / 2$ meter. The presence of water in the shadowed regions at the lunar poles likely comes about from thermal migration caused by solar heating of the lunar surface and the high vapor pressure of volatile species. Water brought to the lunar surface by comets may collect at the poles by a vapor transport mechanism condensing in perpetually cold shaded craters. The presence of water in the lunar soil would be a boon to the exploration program being an extremely valuable in-situ resource for human life support and possibly and more importantly for fuel production. Paul Spudis ${ }^{2}$, one of the project scientists for the Clementine study, referred to a possible lunar ice deposit as possibly,

$$
\text { "... the most valuable piece of real estate in the solar system". }
$$

A literature survey investigating the extraction of lunar water by Duke, et. al. ${ }^{3}$ reviews the most recent and significant work in the area of water extraction on the moon. This paper examined a variety of schemes for ice/water extraction and made recommendations for a practical plan. In-situ extraction using microwaves was mentioned but not in any detail. The only other reference we could find was by Taylor ${ }^{4}$. Among other things, a scheme to use microwaves for water extraction was briefly outlined at the end of this his web article. We know of no significant experimental studies with microwave processing to extract water having been performed by any research group.

The lunar surface has a low thermal conductivity much like good insulation. This strongly disfavors traditional methods of heating. Microwave heating of regolith could be faster and more efficient than any other heating method due to the ability to couple energy into the subsurface volume. Microwaves can penetrate into the soil permitting water removal from deep below the surface with collection above the surface. Microwave processing by the proposed method could permit the extraction of this water without the need to dig, drill, or excavate the lunar surface. The volatile water would be collected on a chilled surface as it percolates from the surface. Multiple synergistic processes make this approach highly efficient. The time and energy of excavation and transport of the regolith is not required and potential damage from raised dust is eliminated. There is no precision machinery involved and few moving parts. Microwave energy can penetrate several feet into the soil past the thin, waterless surface layer. The process is not landing site-specific being insensitive to regolith composition. Finally, the whole process could easily be automated and water extraction rovers could be deployed to roam over the surface of the Moon. This would be very "environmentally friendly" and the least disruptive of the hazardous dust on the surface. Microwave processing could also be used to extract other valuable volatiles from the regolith such as solar wind products and for other uses such as sintering or melting the lunar surface for dust abatement.

By directing the radio frequency energy of a microwave beam into the lunar soil, heating will occur by dielectric absorption selectivity within the regolith particles and the trapped water depending on the 
dielectric properties, temperature, and microwave wavelength. Liquid water has a very high coupling efficiency over two orders of magnitude range of microwave frequencies, but, microwave coupling efficiency to water-ice is much lower than to water. But when the soil is heated the water ice is also heated, causing increasing the water vapor pressure by sublimation.

Normally, basaltic material (composition like lunar mare regolith) has a low absorption (loss tangent) of microwave energy that increases with iron content. Fortunately, the iron and titanium content of the lunar soil (and the stimulant) allows coupling of microwaves as much as 3 orders of magnitude better than many common materials (diamond, fused silica, Teflon, polyethylene). Taylor reported a large microwave coupling efficiency when performing sintering experiments with actual lunar regolith. This was attributed this to nano-phased $\mathrm{Fe}^{0}$ in the surface of grains of lunar soil that enhances heating efficiency when using microwaves to sinter the regolith. Such enhanced coupling of microwave energy to the regolith would aid the heating of the actual lunar soil. The presence of nano-phase metallic $\mathrm{Fe}^{0}$ within the glassy agglutinates of Apollo regolith samples which enhances coupling with microwaves strengthens the case for the use of microwave processing.

\section{EXPERIMENTAL PROCEEDURE}

In anticipation of the possible presence of this water-ice, it is necessary to develop methodology for its extraction. Laboratory experiments are needed for proof of concept tests to determine the efficiency of various methods that could be used to efficiently extract water from the lunar regolith.

The experimental plan was to examine the extent of microwave energy coupling and rate of water extraction from frozen lunar regolith permafrost as a function of microwave power, temperature, concentration of water in the frozen soil, and depth of the soil. The investigation utilizes low cost 2.45 $\mathrm{GHz}$ magnetron sources used in household microwave ovens, so that hardware costs and development time could be kept low.

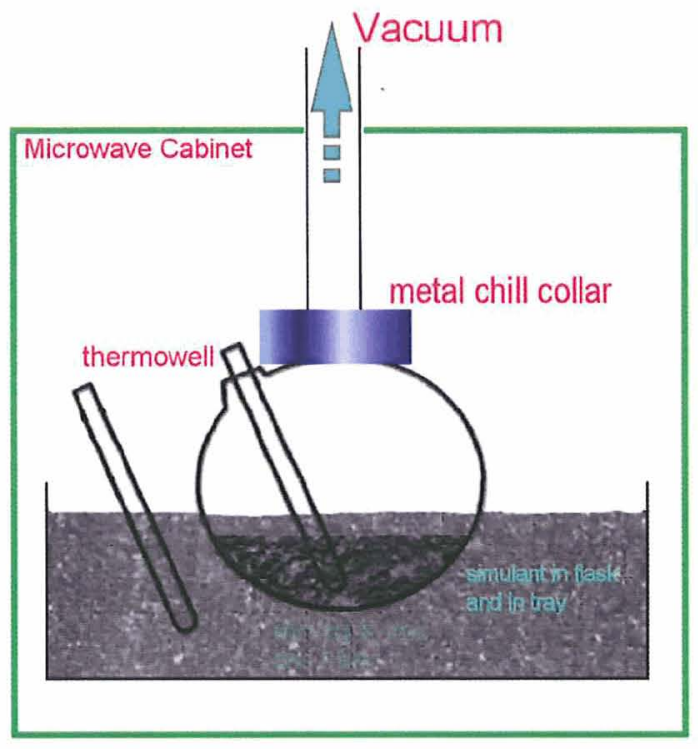

Figure 1. Schematic of one test configuration for microwave extraction of water. 
A simplified schematic for our initial test configuration is shown in Fig. 1. This configuration simulates lunar conditions of temperature and vacuum while microwave processing lunar regolith simulant to extract water. This approach places a microwave-transparent vacuum vessel (fused silica) within a commercial microwave oven cavity. A sealed thermowell in the wall of the vessel permits periodic temperature measurements. Fused silica was found to provide the visibility, mechanical strength, thermal resistance, thermal shock resistance and minimal microwave absorption that made it the ideal vessel material.

An amorphous quartz vessel suitable for evacuation was fabricated by a glass-blower with a throat suitable for connecting to a vacuum line and a well into which a thermocouple can be quickly inserted "into" the heated sample without breaking a vacuum. The thermocouple has to be removed during microwave heating. Lunar regolith simulant, JSC-1, is placed into the quartz vessel and seeded with a given weight percent of water. The quartz vessel is placed into a bed of JSC-1 contained with a polyethylene pan. A vacuum line is passed into a consumer model microwave oven and connected to the top of the quartz vessel. The turbomolecular vacuum pumping station is able to pull a $10^{-7}$ Torr vacuum level.

There is no standard for a lunar regolith water-containing permafrost stimulant. At cryogenic temperatures, any water would be adsorbed water ice (probably amorphous on the moon). It is not likely to be chemically bound to the solid silicate regolith particles. Our lunar regolith permafrost simulants consists of vacuum baking the standard JSC-1 lunar regolith stimulant $(\sim 200 \mathrm{~g})$ in the flask. A measured amount of water $(0.5$ to $2 \%)$ was then injected into the stimulant. The water promptly caused the simulant to clump like kitty litter. After a day of diffusion, the water redistributed itself uniformly.

Soaking the regolith assembly in liquid nitrogen before vacuum is applied eventually brings the regolith bed temperature down to minus $1960^{\circ} \mathrm{C}$. The temperature of the regolith in the vacuum vessel did not get as cold as the regolith bed cooled with liquid nitrogen. This is because of the thermal insulating properties of the regolith soil in vacuum. A suitably chilled surface upstream along the vacuum path permits the water vapor to condense and accumulate on that surface. A glass liquid Nitrogen trap was mounted in line with the pumping station. No measurable water vapor sublimed at these temperatures and our tests showed that water was only released when microwave power was applied. Microwave power was supplied by a $1200 \mathrm{~W}$ consumer kitchen Amana Radarrange (model MW96T). When turned on the microwave energy envelops the entire experimental configuration being absorbed in the supporting simulant bed as well as by the stimulant containing water in the vacuum vessel. The sample-holding materials (fused silica and polyethylene) absorb very little of the microwave energy. Water vapor that was liberated into the vacuum was collected in the liquid nitrogen cold trap. The amount of water condensed provides a measure of the efficiency of the process.

\section{RESULTS AND DISCUSSION}

The experimental facility used for measurements is shown in Figure 2. The parts of the system are identified in the pictures. The individual components are: A fused silica flask containing lunar regolith (JSC-1) permafrost (water containing) stimulant (A), a liquid nitrogen cold trap (B), the turbomolecular vacuum pumping station (C), a JSC-1 lunar simulant bed in a polyethylene tray (D), the $1100 \mathrm{~W}$ microwave oven (E), and the vacuum line to the fused silica flask (F).

This experimental facility was successfully assembled and tested for cryogenic $\left(-196^{\circ} \mathrm{C}\right)$ vacuum $\left(10^{-7}\right.$ Torr $)$ conditions to simulate lunar surface conditions. The frozen water was reasonably stable at these 
temperatures, indicated by the pressure of the system remaining relatively constant without the cold trap. When microwave power was applied, the temperature of the regolith simulant increased, the pressure in the vacuum system increased, and water vapor was condensed into the external cold trap.

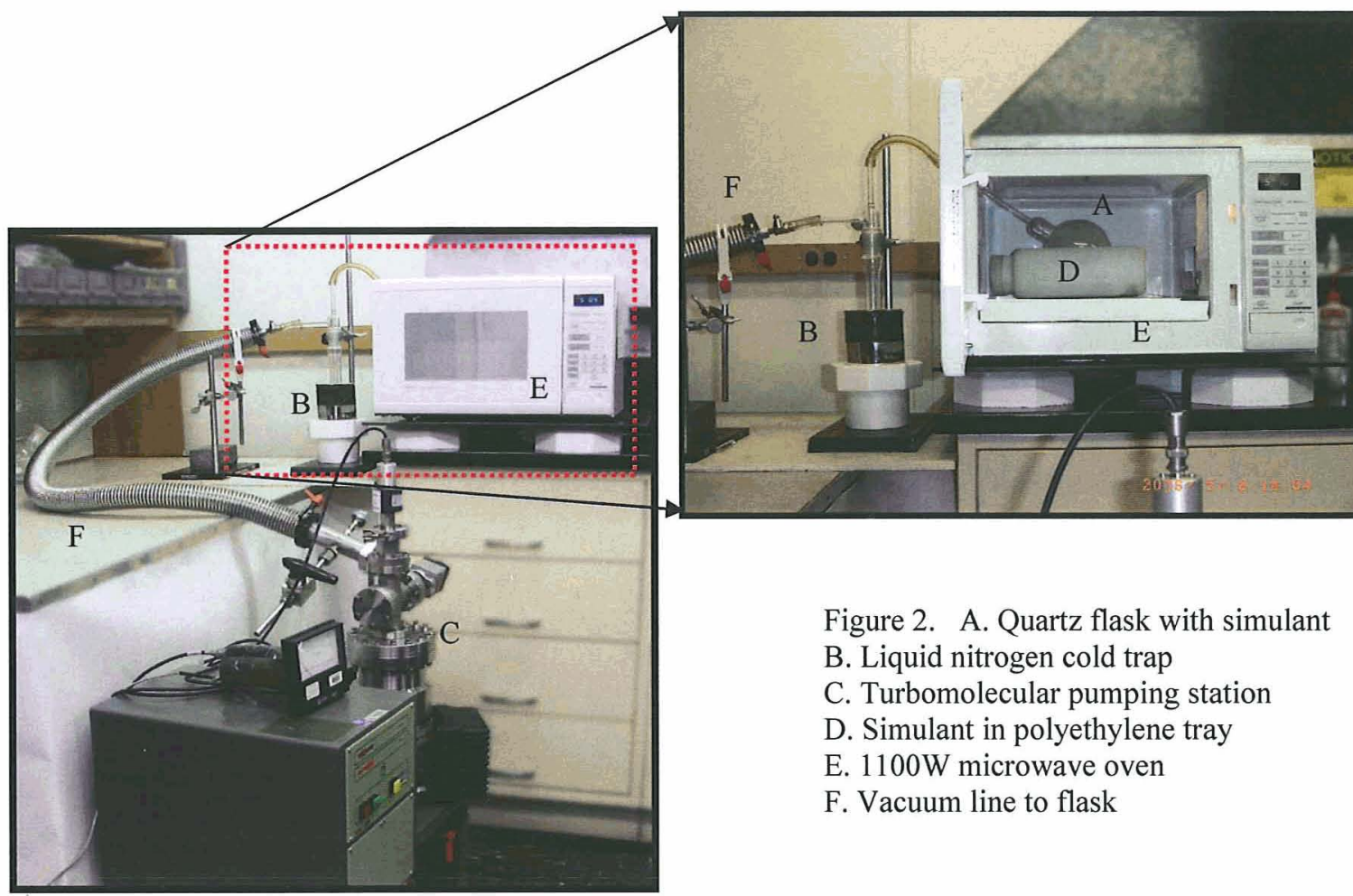

At room temperature, the moist simulant had a clumping behavior and tended not to slump when poured into a pile. The behavior is reminiscent of wet sand. A $2 \%$ mixture was sealed in the vessel and permitted to disperse throughout the regolith for a couple of days. It was then cooled with liquid nitrogen. This same mixture flowed as readily as salt or very dry sand when the temperature was well below freezing $\left(0^{\circ} \mathrm{C}\right)$. Simulant with up to $2 \%$ water behaves as a dry powder once the temperature drops well below freezing. This contradicts some suggestions that the lunar permafrost could be a frozen monolithic solid. Naturally, the behavior of the soil with frozen water depends on the amount of water present.

Cryogenic cooling of the regolith was a slow process. The regolith was saturated with liquid nitrogen for half an hour or more. The regolith in the vacuum vessel never did reach the same temperature as the regolith bed because of the low thermal conductivity. The Lunar Sourcebook ${ }^{5}$ contains a description of thermal measurements of the regolith using a special probe buried into soil. From those Apollo missions $(15,16 \& 17)$ where thermal conductivity was measured, the value of $150 \times 10^{-6} \mathrm{~W} / \mathrm{cm} \mathrm{K}$ was determined for lunar regolith at $1 \mathrm{~m}$ depth at the Apollo 15 site. At the Apollo 17 site, a value of 172 to $295 \times 10^{-6}$ $\mathrm{W} / \mathrm{cm} \mathrm{K}$ was measured and was attributed to the higher regolith density. In order to obtain a sense of this, other materials were searched with similar thermal conductivities. The only material that came low enough was silica aerogel (a super-insulation) with a value of $80 \times 10^{-6} \mathrm{~W} / \mathrm{cm} \mathrm{K}$. This surprising result places far 
more importance on design for heat transfer when processing regolith. We can only assume the thermal conductivity of the stimulant in a vacuum is close to that of the lunar soil.

The regolith temperatures were measured with thermocouples from the interior of the simulant volume. For each measurement taken, a few seconds were allowed for the thermocouple to equilibrate with the thermowell, and after that point, even for a minute or two, the temperature remained constant. With the high level of insulation offered by this simulant, there was little error introduced by interrupting the heating cycle in order to take the measurement.

The dielectric properties of materials change with temperature such that microwave absorption increases dramatically with temperature above a critical temperature. This point is typically 400 degrees $\mathrm{C}$ or more. However, there are smaller variances in the dielectric properties at lower temperatures as well. There is little data in the literature of the dielectric properties of common materials at microwave frequencies (and certainly none of simulant) at cryogenic temperatures.

Since the temperature dependence of the dielectric properties of the lunar regolith stimulant is not known, we were unsure how the simulant would respond when heated with microwaves while at liquid nitrogen temperatures. Prior to adding water to the JSC-1 simulant, the heating of the stimulant was tested. Although a simple heating experiment doesn't provide dielectric property data, it can demonstrate the feasibility of working at those temperatures and shows the reaction to microwaves at $2.45 \mathrm{GHz}$. In all our tests with JSC-1 and JSC-1AF simulants heated from cryogenic temperatures to $100^{\circ} \mathrm{C}$, the rate of temperature increase over heating time was linear. This result implies the coupling efficiency was nearly constant over this temperature range. In turn, this indicates the dielectric constant does not significantly change over this nearly 300 degree span. This is consistent with the lunar dielectric permittivity measurements that show a constant value below $300^{\circ} \mathrm{C}$ at radio frequencies. It should be noted that (from

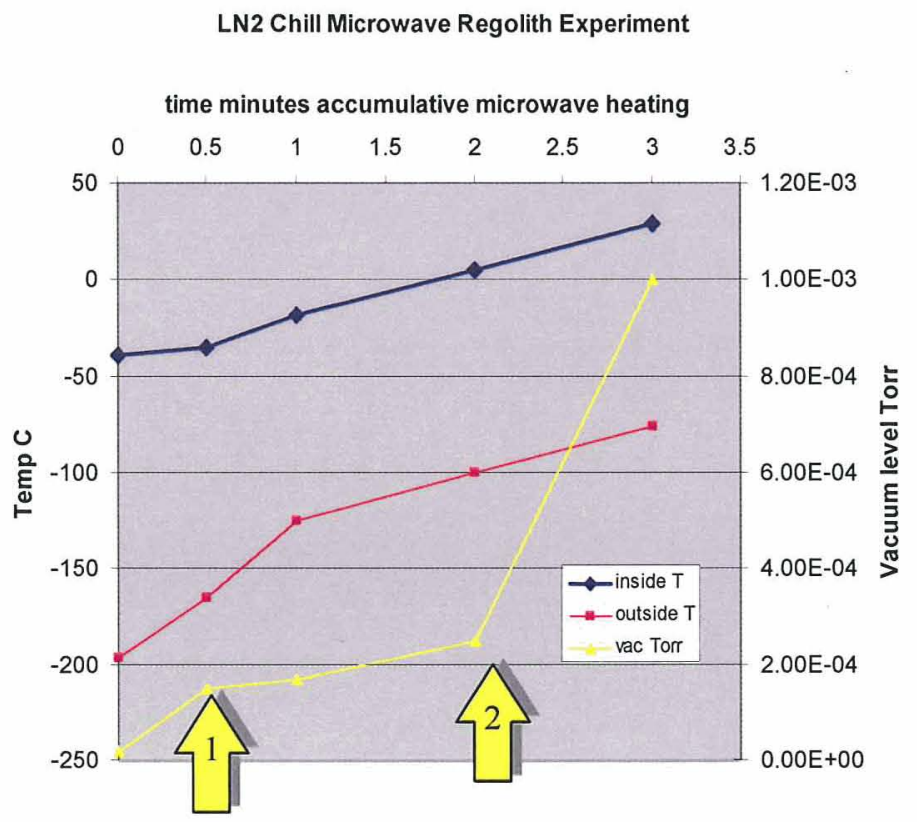

Figure 3. Cryogenic cooled lunar regolith permafrost stimulant. Temperature rise vs. microwave heating time (red \& blue lines).

Water vapor pressure level increase with microwave heating time (yellow line). Some water vapor appears immediately upon microwave power-up (arrow 1).

Water vapor pressure shoots up when stimulant temperature reaches the freezing point (arrow 2). 
our experiments) the lunar simulant couples well to microwave energy and one might expect actual lunar regolith to couple even better. This heating of the simulant is not compromised by cryogenic cooling over the range tested.

The first experiment with our lunar regolith permafrost stimulant was performed without the cold trap. The purpose was to evaluate the effect of temperature of the stimulant on the rate of sublimation and water vapor production. Figure 3 shows a graph of lunar simulant temperatures and the vacuum level as function of microwave process time. The maximum microwave power was applied step wise in 30 second intervals with temperature measurements made between each step. The temperature of the regolith simulant from the thermowell thermocouple measurement of the stimulant under vacuum is the blue line while the red line is from a thermocouple measurement in the regolith bed surrounding the fused silica vacuum vessel. The simulant temperature rises proportionately with accumulated power absorbed over the temperature range.

Water vapor came off immediately upon microwave power-up, and effectively none until the initial powerup (arrow 1). With subsequent microwave heating steps, the water vapor pressure (yellow trace) rises with temperature until there is a sudden increase when the regolith stimulant permafrost (blue line) is heated to above $0^{\circ} \mathrm{C}$ (arrow 2).

One last experiment evaluated the amount of water extracted from the regolith permafrost stimulant and the efficiency of a cold trap to recover the water. In this experiment the cold trap was filled with liquid nitrogen and the microwave was again run at full power. As heating progressed, the pressure increased due to some water vapor not being trapped. After a total of 5 minutes of heating and subsequent weighing of the stimulant and the cold trap, it was determined that $85 \%$ of the water had been extracted from the regolith. And of that, $99 \%$ of the sublimed water removed from the regolith had been captured with the cold trap. This verifies both high levels of extraction and water collection with this microwave volatile extraction method.

\section{FUTURE PLANS}

In the next step for this project, a microwave vacuum chamber will need to be assembled to investigate the extraction of water from different depths within the lunar stimulant. For this work, large vacuum gloveboxes at MSFC could be used to place the microwave unit into the vacuum chamber and microwaves delivered downward into a deep container containing lunar stimulant. Direct real time temperature measurement is not possible within the $2.45 \mathrm{GHz}$ microwave with traditional temperature measuring instrumentation such as metal thermocouples and Pt resistance thermometers. Experiments are being developed with an optical fiber thermometer from Luxtron which can be used within the microwave environment. A cold trap suitable for use on the moon needs to be designed and evaluated and used to quantify the rate of water removal of water removal.

There are open issues regarding the most suitable microwave frequency to use. This is especially important for penetrating into the surface of the moon to extract water and cannot be solved by calculations as to penetration depth differences versus heating efficiency. On one hand, lower frequencies penetrate very deeply, because absorption is low thus not contributing to heating. On the other hand, there is an exponential decay of microwave absorption from the surface inwards. This surface heating effect increases as frequency increases. Ultimately we need data from the moon as to how deep and where the water is. For extraction efficiency, we want to reduce non-productive heating of regolith. This may also be frequency dependent. 
Ultimately we would like to have the opportunity to develop an RLEP experiment. Magnetron microwave sources producing kilowatts of power might be used for a practical water extraction robot. But, for a lunar lander rover experiment, to find and verify the extraction of water, light weight and low power consumption is in order. For the one or two hundred watts likely to be available for a lunar lander experiment, a fully solid-state electronic source for the microwaves could be used. This offers miniaturization, low mass, low power and simpler thermal control. These lower power solid-state microwave electronic sources need to be developed and tested, with the goal of a demonstration lunar lander experiment. There is the need to perform design studies of a prototype of a space qualified lunar water detection and extraction device.

\section{CONCLUSIONS}

A lunar stimulant test bed facility was tested for cryogenic $\left(-196^{\circ} \mathrm{C}\right)$ vacuum $(10-6$ torr $)$ conditions. Water extraction from lunar regolith permafrost stimulant with water-ice $(0.5$ to $2 \%)$ was tested with a consumer microwave oven under vacuum cryogenic conditions. This proof of principal demonstration of significant water removal well below $0^{\circ} \mathrm{C}$ was successful. The lunar permafrost simulant couples well to microwave energy and one might expect lunar regolith to couple even better. This coupling to simulant was not compromised by cryogenic cooling over the range tested which was representative of Lunar temperatures. After a short 5 minutes of heating, at least $85 \%$ of the water was removed from the lunar regolith stimulant and $99 \%$ of that water was captured in a cold trap.

\section{ACKNOWLEDGMENTS}

The authors would like to thank Carole McLemore VP33 in the In-Situ Resource Utilization and In-Space Fabrication and Repair group at the Marshall Space Flight Center for support and funding of this research project.

\section{REFERENCES}

1. Feldman, W. C.; Maurice, S.; Lawrence, D. J.; Little, R. C.; Lawson, S. L.; Gasnault, O.; Wiens, R. C.; Barraclough, B. L.; Elphic, R. C.; Prettyman, T. H.; Steinberg, J. T.; Binder, A. B.; "Evidence for water ice near the lunar poles"; Journal of Geophysical Research, Volume 106, Issue E10, p. 23231-23252

2. Bussey, B. and Spudis, P.; "Small Spacecraft Exploration of the Moon"; 54th International Astronautical Congress of the International Astronautical Federation, the International Academy of Astronautics, and the International Institute of Space Law; 29 September - 3 October 2003, Bremen, Germany, IAC-03-IAA.11.3.04

3. Duke, M. et. Al. (1999), "Lunar Polar Ice Methods for Mining the New Resource for Exploration" presented at the Space Resources Utilization Roundtable, October 27-29, 1999 at the Colorado School of Mines in Golden, CO.

4. Taylor, L. and Meek, T. "Microwave Sintering of Lunar Soil: Properties, Theory, and Practice," J. Aerospace Eng. 18:188-196(2005). $\quad$ www.lpi.usra.edu/lunar knowledge/LTaylor.pdf

5. Lunar Sourcebook - a User's Guide to the Moon, G.H. Heiken, D.T. Vaniman, and B.M. French, Eds., Cambridge University Press, Cambridge, (1991) 736 pp 